\title{
Network Flow Models for Intraday Personnel Scheduling Problems
}

\author{
Peter Brucker ${ }^{1}$ and Rong $\mathrm{Qu}^{2}$ \\ ${ }^{1}$ Universität Osnabrück, Albrechtstr. 28a, 49069 Osnabrück, Germany \\ e-mail:pbrucker@uni-osnabrueck.de \\ ${ }^{2}$ Automated Scheduling, Optimization and Planning (ASAP) Group, School of \\ Computer Science, University of Nottingham, NG8 1BB, UK \\ e-mail:rxq@cs.nott.ac.uk
}

\begin{abstract}
Personnel scheduling problems can be decomposed into two stages. In the first stage for each employee the working days have to be fixed. In the second stage for each day of the planning period an intraday scheduling problem has to be solved. It consists of the assignment of shifts to the employees who have to work on the day and for each working period of an employee a task assignment such that the demand of all tasks for personnel is covered. In Robinson et al. [10], the intraday problem has been formulated as a maximum flow problem. The assumptions are that, employees are qualified for all tasks, their shifts are given, and they are allowed to change tasks during the day.

In this work, we extend the network flow model to cover the case where not all employees are qualified to perform all tasks. The model is further extended to be able to calculate shifts of employees for the given day, assuming that an earliest starting time, a latest finishing time, and a minimal working time are given. Labour cost can be also taken into account by solving a minimum cost network flow problem.
\end{abstract}

KEYWORDS: personnel scheduling, assignment problem, network flows

\section{Introduction}

Personnel scheduling problems represent an important operational management activity in many organizations [8]. The problem often consists of two steps. The first step usually determines the assignment of working days to employees. The second step concerns the assignment of tasks to be done by the employees on each working day of the employee to cover the staffing requirement. In [7], the staff scheduling and rostering process is defined as starting with the determination of staffing requirement, where demand is modeled based on duty requirements. For example, in call center [9] and supermarket staff scheduling, the staffing level is quite often modeled based on hourly intervals each day, over several weeks horizon. In driver scheduling, staffing is determined based on tasks with a starting time and duration, within certain time windows and associated to transport timetables. In nurse rostering [3], different types of shift are defined based on the 24/7 service requirement over different days of the scheduling period.

Quite often characters of personnel scheduling problems have to be 
considered to develop specific mathematical models in different areas [8]. This is the reason why there is a lack of general models in the personnel scheduling problem [5]. In this work we extend the network flow model presented by Robinson et al. [10] by concerning the additional preemption requirement, aiming to further raise the generality of personnel scheduling models.

A class of personnel scheduling problems can be formulated as follows.

There is a planning horizon consisting of a number of consecutive days. Associated with each day is a set of periods in which certain tasks have to be performed. For each period of a day and for each task which has to be performed in this period, employees are needed.

The planning horizon has to be divided into working days and rest days for each employee. A shift has to be assigned to each working day of an employee. Shifts consist of a set of working periods possibly interrupted by breaks and idle times which are part of the shift. Working periods in the shift often vary between employees due to their different contract constraints. Some personnel scheduling problems may have common standard working periods. They usually also depend on policies and regulations of the companies.

For each employee there is a set of tasks he can be assigned to.

A working pattern is defined by the set of working days and for each working day a shift. A working pattern is feasible for an employee if it satisfies a number of constraints.

One has to assign

- to each employee a feasible working pattern, and

- to each working period of this pattern tasks to be performed by the employee.

This has to be done in such a way that

- all tasks can be performed (i.e. the demand of tasks for employees is satisfied), and

- corresponding costs are minimized.

Depending on their characteristics, personnel scheduling problems can be decomposed in some common ways [7,9]. In most problem scenarios, demand modeling could be separated naturally, leaving the scheduling of shifts and task assignment relatively independent. This decomposition results into a number of sub-problems and some of them become tractable [2].

In this work, following the decomposition by Robinson et al. [10], the model has two levels which we denote by days scheduling and intraday scheduling level. At the days level one has to assign working days to employees while at the intraday level for each employee working on the day one has to assign a shift and to each working period of this shift tasks for which the employee is qualified. In call center [9] and supermarket staff scheduling, this means firstly scheduling employees to shifts of working days, and then to tasks during the day depending on the demand. In nurse rostering [3], nurses are 
scheduled to working days, but there is no need to schedule tasks, i.e. nurses are usually required to work during the pre-defined shift of certain duration on the day. A benchmark dataset web site [4] provides a collection of such instances from around the world.

One can differentiate between preemptive and non-preemptive problems. Preemption means a task can be temporarily interrupted and resumed later. Furthermore, in a preemptive problem employees may change the working place during a shift. This is not allowed in non-preemptive versions.

In the literature some network flow models have been discussed for personnel scheduling problems. A network flow model for a special non-preemptive personnel scheduling problem is discussed in [11]. Robinson et al. [10] formulated the intraday problem as a maximum flow problem under the following assumptions: employees are qualified for all tasks, their shifts are given, and they are allowed to change tasks during the day.

We show that the network flow model can be extended to cover the case in which employees are not qualified to perform all tasks. Further extensions allow to calculate shifts of employees for the given day under the assumption that an earliest starting time and a latest finishing time as well as a minimal working time are given. Also labour cost can be taken into account by solving a minimum cost network flow problem.

This paper is organized as follows. The maximum flow model of Robinson et al. [10] is presented in Section 2, followed by the extended network flow model in Section 3. In Section 4 we present further extensions concerning demand and supply aspects of the network model we build in Section 3. The last section contains concluding remarks.

\section{The Maximum Flow Formulation of Robinson et al. [10]}

The intraday personnel scheduling problem of Robinson et al. [10] can be described as follows.

Each day a subset of employees is available. Each employee $e$ working on a fixed day is available during some time window $\left[S_{e}, F_{e}[\right.$. A shift of employee $e$ is a time interval $\left[V_{e}, W_{e}\right.$ [with $S_{e} \leq V_{e} \leq W_{e} \leq F_{e}$ and $W_{e}-V_{e} \geq$ $m_{e}$ where $m_{e}$ is a given minimal shift length. During each period within a shift the employee performs a task, or has a (long or short) break, or is idle. When scheduling breaks, there are minimal and maximal restrictions on the period between

- the starting time $V_{e}$ of the shift and the start of the first break;

- the end of the last break and the finish time $W_{e}$ of the shift;

- the end of one break and start of the next.

All breaks are not preemptive.

There are $n$ tasks $j=1, \ldots, n$. Each task $j$ has a duration $p_{j}$ and must be processed by exactly one employee at a time within a time window $\left[R_{j}, D_{j}\right.$ [ with 
$D_{j}-R_{j} \geq p_{j}$. A task can be split into portions, and performed by different employees. Preemption is allowed, i.e. different employees may perform a task and an employee may switch different tasks on a day. Also interruption and later resumption of a task is possible. However, the total processing of task $j$ must be equal to $p_{j}$.

Each employee can be assigned to any task.

One has to assign feasible shifts to the employees and for each shift to assign tasks to its active periods such that

- the duration of each task is covered within its time window, and

- the total labor cost is minimized.

Under the assumption that for each employee a shift has been fixed the problem can be formulated as a maximum flow problem with the following data.

Blocks are defined as the maximal sets of consecutive working periods of a shift. Let $T$ be the set of all $R_{j^{-}}$and $D_{j^{-}}$values of task $j$, and the starting and finishing times of the blocks for all employees working on the day. Denote by $t_{1}<t_{2}<\ldots<t_{s}$ the ordered sequence of all elements in $T$.

The network $(V, A)$ can be constructed as follows. The set $V$ of nodes consists of

- task nodes $j=1, \ldots, n$,

- interval nodes $\left[t_{i}, t_{i+1}[(i=1, \ldots, s-1)\right.$, and

- a source $s$ and a sink $t$.

There are three different types of directed arcs:

- $\operatorname{arcs}(s, j)$ with upper capacity $p_{j}$,

- $\operatorname{arcs}\left(\left[t_{i}, t_{i+1}[, t)\right.\right.$ with upper capacity $\left(t_{i+1}-t_{i}\right) N_{i}$ where $N_{i}$ is the number of employees available in time period $\left[t_{i}, t_{i+1}[\right.$,

- there is an arc between a task node $\mathrm{j}$ and an interval node $\left[t_{i}, t_{i+1}[\right.$ if and only if $\left[t_{i}, t_{i+1}\left[\subseteq\left[R_{j}, D_{j}\left[\right.\right.\right.\right.$. The upper capacity of this arc is $\left(t_{i+1}-t_{i}\right)$.

The network is shown in Figure 1.

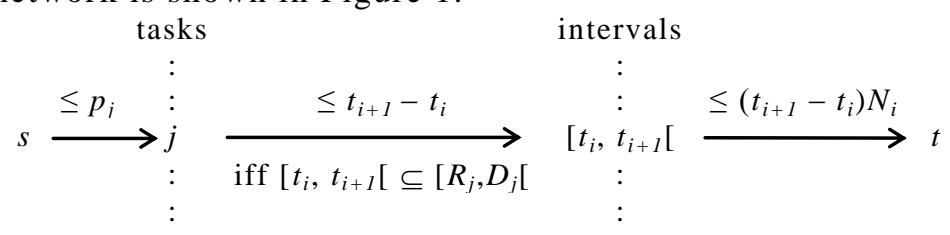

Figure 1: Network for the assignment of tasks to employees

A flow in an arc $\left(j,\left[t_{i}, t_{i+1}[)\right.\right.$ may be interpreted as working time assigned to task $j$ in the interval $\left[t_{i}, t_{i+1}[\right.$. There exists a feasible task assignment if and only if the value of a maximum flow is equal to $\sum_{j=1}^{n} p_{j}$.

If there is a maximal flow with this property then in each task node $j$ the processing time $p_{j}$ is distributed to the time intervals $\left[t_{i}, t_{i+1}[\right.$ in which $j$ can be processed and the total time in which $j$ is processed in $\left[t_{i}, t_{i+1}\right.$ [ cannot exceed $t_{i+1}-t_{i}$. Furthermore, due to the flow-balance constraints in the interval 
nodes $\left[t_{i}, t_{i+1}\right.$ [ the sum of these processing times cannot exceed $\left(t_{i+1}-t_{i}\right) N_{i}$. It is well known (see e.g. [1] P.108) that under these conditions it is possible to process the parts of tasks assigned to $\left[t_{i}, t_{i+1}\right.$ [ by $N_{i}$ employees if preemption is allowed, i.e. tasks can be interrupted.

Robinson et al. [10] describe a tabu search heuristic to schedule the set of shifts for the employees for a given time horizon of several days. The aim is to search for schedules where shifts cover the demand of all tasks. Such shifts are called feasible. Feasibility can be checked and corresponding task assignments can be calculated by solving a maximum flow problem for each day. The objective is to find feasible shifts with minimum labor costs. In the tabu search, different types of moves are made to change a shift at a time for an employee.

The assumption that each employee can be assigned to any task is not always realistic. Therefore the model will be extended in the next section.

\section{An Extended Network Flow Model}

In this and later sections the assumption that employee $e$ can perform only tasks $j \in Q_{e} \subseteq\{1, \ldots, n\}$ is added. For example, in nurse rostering and call center scheduling, certain skills of employees must be taken into account when scheduling the tasks. A network which takes care of these additional constraints can be described as follows.

Again $t_{1}<t_{2}<\ldots<t_{s}$ are the time instances where the time dependant data are changing. The set of nodes of the network consists of

- task nodes $j=1, \ldots, n$,

- interval-task nodes $\left[t_{i}, t_{i+1}\left[_{j}\right.\right.$ for all intervals $\left[t_{i}, t_{i+1}\left[\right.\right.$ with $\left[t_{i}, t_{i+1}\left[_{j}\right.\right.$ $\subseteq\left[R_{j}, D_{j}[\right.$,

- interval-employee nodes $\left[t_{i}, t_{i+1}\left[e\right.\right.$ for all working intervals $\left[t_{i}, t_{i+1}[\right.$ of employee $e$, and

- a source $s$ and a sink $t$.

There are four different types of arcs:

- $\operatorname{arcs}(s, j)$ with upper capacity $p_{j}$,

- $\operatorname{arcs}\left(j,\left[t_{i}, t_{i+1}\left[_{j}\right)\right.\right.$ with upper capacity $t_{i+1}-t_{i}$,

- $\operatorname{arcs}\left(\left[t_{i}, t_{i+1}\left[_{j},\left[t_{i}, t_{i+1}\left[_{e}\right)\right.\right.\right.\right.$ for $j \in Q_{e}$, and

- $\operatorname{arcs}\left(\left[t_{i}, t_{i+1}[e, t)\right.\right.$ with upper capacity $t_{i+1}-t_{i}$.

The network is shown in Figure 2. A flow in an arc $\left(\left[t_{i}, t_{i+1}\left[j,\left[t_{i}, t_{i+1}[e)\right.\right.\right.\right.$ may be interpreted as the number of time units employee $e$ is assigned to task $j$ within the time interval $\left[t_{i}, t_{i+1}\right.$ [. The flow conservation constraint for node $\left[t_{i}, t_{i+1}\left[_{j}\right.\right.$ distributes the time spent on task $j$ in $\left[t_{i}, t_{i+1}[\right.$ among employees which are qualified to do task $j$. The flow conservation constraint for node $\left[t_{i}, t_{i+1}\right.$ [e limits the workload of employee $e$ in $\left[t_{i}, t_{i+1}\left[\right.\right.$ by $t_{i+1}-t_{i}$. There exists a feasible assignment of employees to tasks if and only if the maximum flow is equal to $\sum_{j=1}^{n} p_{j}$. 


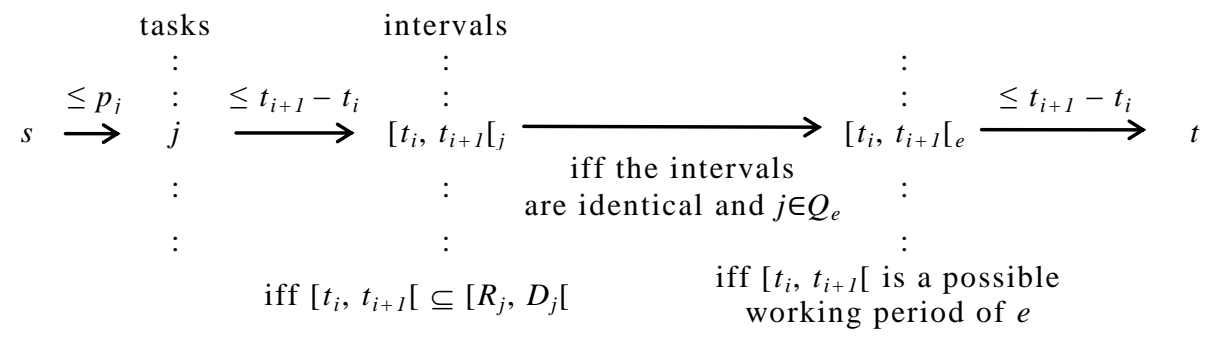

Figure 2: Extended network

The procedure is illustrated by the following example with two employees and three tasks.

Example 1 Consider a problem with the following data. Notice that in the time interval $\left[2,3\left[\right.\right.$ employee $e_{1}$ has a break.

$\begin{array}{cccc}\operatorname{task} j & 1 & 2 & 3 \\ R_{j} & 0 & 3 & 4 \\ D_{j} & 6 & 7 & 6 \\ p_{j} & 4 & 2 & 2\end{array}$

$$
\begin{array}{cc}
\text { employee } e_{i} & \text { shift } \\
e_{1} & {[0,2[,[3,6[} \\
e_{2} & {[3,7[}
\end{array}
$$

The corresponding network with a solution is presented in Figure 3. Figure 4 shows the Gantt chart of the solution. The relevant $t_{i}$ values are $0,2,3,4,6,7$. Employee $e_{2}$ is idle in period [3,4[. Note that in Figure 3, we keep the arc between task 1 and task interval $\left[2,3\left[_{1}\right.\right.$, even there is no flow on the arc in this specific example. In general network flow techniques, the existence of such arcs can be usually processed automatically; therefore we keep this arc to present the network flow model for general cases.

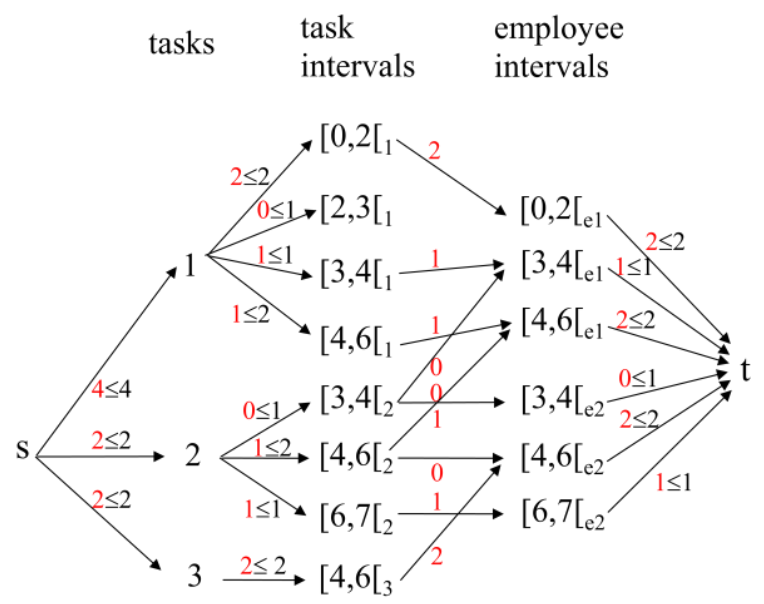

Figure 3: Network flow of Example 1 


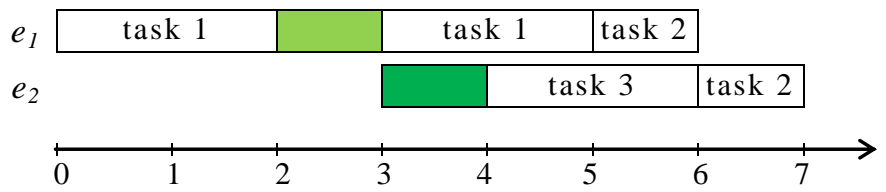

Figure 4: Gantt chart of the solution for Example 1

\section{Further Extensions}

The model introduced in the previous section can be extended at the demand side and/or the supply side. Possible extensions will be discussed in this section.

\subsection{Extensions at the Demand Side}

Instead of forcing the processing time of each task $j$ to be equal to $p_{j}$ by solving a corresponding maximum flow problem, it is possible to enforce the constraint $L P_{j} \leq p_{j} \leq U P_{j}$ by the lower bound $L P_{j}$ and the upper bound $U P_{j}$ for the flow in the arc $(s, j)$. In this case one has to find a feasible solution. If additionally costs are assigned to the arcs $\left(\left[t_{i}, t_{i+1}[e, t)\right.\right.$ one could minimize labour costs by solving a corresponding minimum cost network flow problem.

Another option is to replace

by

$$
s \stackrel{\leq p_{j}}{\longrightarrow} j \stackrel{\leq t_{i+1}-t_{i}}{\longrightarrow}\left[t_{i}, t_{i+1}\left[{ }_{j}\right.\right.
$$

$$
s \stackrel{\leq p_{i j}\left(t_{i+1}-t_{i}\right)}{\longrightarrow}\left[t_{i}, t_{i+1}\left[_{j}\right.\right.
$$

Where $p_{i j}$ is the number of employees needed for task $j$ in the time interval [ $t_{i}$, $t_{i+1}$ [. Again one has to solve a maximum flow problem to cover the demand. Also by lower and upper bounds on the $\operatorname{arcs}\left(s,\left[t_{i}, t_{i+1}\left[_{j}\right)\right.\right.$ the constraints $L D_{i j}\left(t_{i+1}-t_{i}\right) \leq p_{i j}\left(t_{i+1}-t_{i}\right) \leq L D_{i j}\left(t_{i+1}-t_{i}\right)$ can be enforced.

\subsection{Extensions at the Supply Side}

Instead of fixing the shift of employee $e$ in advance one could fix only the availability interval $\left[S_{e}, F_{e}\left[\right.\right.$ and a minimal working time $m_{e}$ for employee $e$. Then shifts for the employees which cover the demand of tasks can be calculated. To achieve this one has to replace

by

$$
\left[t_{i}, t_{i+1}\left[_{e} \stackrel{\leq t_{i+1}-t_{i}}{\longrightarrow} t\right.\right.
$$

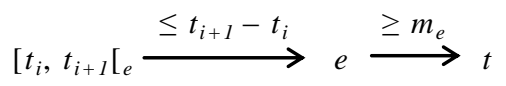

Due to node $e$ and $\operatorname{arc}(e, t)$ the total working time of employee $e$ cannot be smaller than $m_{e}$. 


\subsection{Combined Extensions}

The extensions at the demand and supply side can be combined. A possible combination is shown in Figure 5 where $\Delta_{i}:=t_{i+1}-t_{i}$. A feasible network flow solution corresponds to a feasible shift and task assignment. Also overtime costs can be taken into account by assigning these overtime costs to the arcs $(e, t)$, zero costs to all other arcs, and by solving the corresponding minimum cost network flow problem.

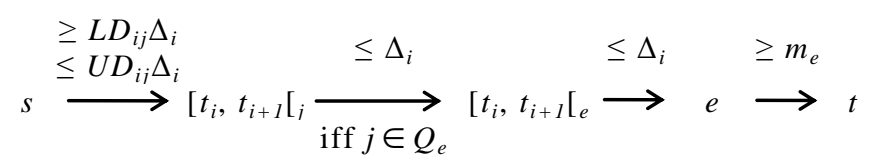

Figure 5: Combined extensions

\section{Concluding Remarks}

In this note we have shown that the problem of assigning shifts to employees, and employees to tasks to cover the demand, can be efficiently solved by network flow algorithms if preemption is allowed, even if employees are not qualified for all tasks. The network flow model can be further extended to concern different requirements in specific personnel scheduling problems.

The extended network flow model can be useful to detect feasible working day patterns. Based on the network flow model, heuristics can be then further applied to schedule shifts of employees when preemption is and is not allowed in personnel scheduling problems. For example, in Robinson et al. [10], a tabu search has been proposed to search for schedules for the personnel scheduling problem. The network model is used to check the eligibility of the proposed schedule. That is to check whether it completes all tasks. Further investigations could be carried out to systematically study the efficiency and effectiveness of integrating the network flow models in advanced meta-heuristics which are designed to address different personnel scheduling problems with specific characteristics.

However, a side effect is that employees have to switch between tasks (working places) during their shifts. These switches depend on the constraints under which shifts are calculated and may be unavoidable. In connection with this the following working place change minimization (WPCM-) problem is of interest: Assume that shifts have been assigned to all employees working on a given day. Then we call a task assignment for these employees feasible if the demand of all tasks for employees is covered. Find a feasible assignment which minimizes the number of working place changes of employees.

In [2] it has been shown that the WPCM-problem is NP-hard if possible shifts for $e$ have the form $\left[t, t+p_{e}\left[\left(t=0, \ldots, P-p_{e}\right)\right.\right.$ where $P$ is the number of working periods of the day. The complexity of the WPCM-problem for other ways of shift assignments is unknown. 
Based on the presented network flow models, extended investigations will be carried out in our future work to systematically develop and study heuristic algorithms which assign feasible shifts to employees and construct (directly) preemptive schedules taking care of working place changes (e.g. by constructing good shifts). Numerical results will be reported and analysed on solving real world problems. Such extensive investigation, which is out of the scope of this paper, will be carried out based on the network flow model presented in this work.

\section{References}

[1] Brucker, P. (2007), Scheduling algorithms. Springer, Berlin.

[2] Brucker, P., Qu, R., Burke, E. (2011). Personnel scheduling: Models and complexity. European Journal of Operational Research, 210(3): 467-473.

[3] Burke, E., De Causmaecker, P., Vanden Berghe, G., Van Landeghem, H. (2004). The state of the art of nurse rostering. Journal of Scheduling, 7(6): 441-499.

[4] Curtois, T. Employee Scheduling Benchmark Data Sets. http://www.cs.nott.ac.uk/ tec/NRP/

[5] De Causmaecker, P., Vanden Berghe, G. (2012). Towards a reference model for timetabling and rostering. Annals of Operations Research, DOI: 10.1007/s10479-010-0721-2.

[6] Eitzen, G., Panton, D., Mills, G. (2004). Multi-Skilled workforce optimisation. Annals of Operations Research. 127(1-4): 359-372.

[7] Ernst, A., Jiang, H., Krishnamoorthy, M., Sier, D. (2004). Staff scheduling and rostering: A review of applications, methods and models. European Journal of Operational Research. 153(1): 3-27.

[8] Ernst, A.T., Jiang, H., Krishnamoorthy, M., Owens, B. and Sier, D. (2004). An annotated bibliography of personnel scheduling and rostering. Annals of Operations Research, 127(1-4): 21-144.

[9] Ertogral K. and Bamuqabel B. (2008). Developing staff schedules for a bilingual telecommunication call center with flexible workers. Computers \& Industrial Engineering, 54(1): 118-127.

[10] Robinson, R., Sorli, R., Zinder, Y. (2005). Personnel scheduling with time windows and preemptive tasks. In: E. Burke and M. Trick (editors), Proceedings of the 5th International Conference on the Practice and Theory of Automated Timetabling, 18th August - 20th August 2004, Pittsburgh, PA USA: 561-566.

[11] Segal, M. (1974). The operator scheduling problem: A network flow approach. Operations Research, 22: 808-823. 